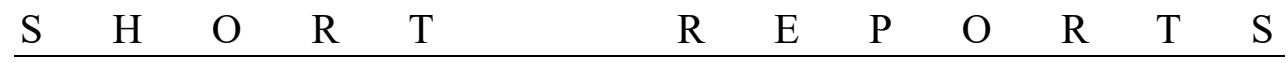

ROCZNIKI PSYCHOLOGICZNE/ANNALS OF PSYCHOLOGY

2020, XXIII, 2, 191-200

DOI: http://dx.doi.org/10.18290/rpsych20232-5

MAGDALENA SZUBIELSKA

MARCIN WOJTASIŃSKI

KATARZYNA BIEDROŃ

MATEUSZ BOBEL

NATALIA CHUDZIAK

Institute of Psychology

John Paul II Catholic University of Lublin, Poland

\title{
CANONICAL SIZE FOR REAL-WORLD OBJECTS IN DRAWINGS PERFORMED UNDER HAPTIC CONTROL
}

To date canonical size for physical objects has been exclusively investigated in the visual domain and termed canonical visual size. As the visual and haptic modalities are interconnected in object processing, we have investigated if canonical size occurs in the tactile domain, namely, in embossed drawings made by sighted adults when blindfolded. 17 participants were asked to draw 16 objects of 8 different ranks of physical size. In the visual domain, they drew on sheets of paper, and in the tactile domain, they drew (when blindfolded) on special plastic sheets for embossed graphics haptically controlling the performance with hands. In both the visual and the tactile domain the size of drawings increased linearly with the logarithm of the physical size of real-world objects indicating occurrence of canonical size effect in both domains. Our findings demonstrated that canonical size is not only visual in character but that it is also revealed in a haptic drawing task. It suggests that spatial images (at least visual and tactile) are shared instead of being unimodal in nature.

Keywords: canonical size; visual domain; tactile domain; physical size; drawings.

Correspondence concerning this article should be addressed to MAGDALENA SzUBIELSKA, PhD, Institute of Psychology, John Paul II Catholic University of Lublin, Al. Racławickie 14, 20-950 Lublin, Poland; email: magdasz@kul.pl; ORCID: https://orcid.org/0000-0002-8437-0871; MARCIN WOJTASIŃSKI, ORCID: https://orcid.org/0000-0001-7581-5659; KATARZYNA BIEDROŃ, ORCID: https://orcid.org/0000-0002-1326-5767; MATEUSZ BOBEL, ORCID: https://orcid.org/00000002-5497-229X; NATALIA CHUDZIAK, ORCID: https://orcid.org/0000-0002-0743-9580. 


\section{INTRODUCTION}

A couple of studies - both behavioral (Konkle \& Oliva, 2011, 2012a; Long et al., 2016) and neuroimaging (Konkle \& Oliva, 2012b) - have shown that information on physical size is part of the mental representation of objects. Konkle and Oliva (2011) ascertained that adults, based on their knowledge of real-world objects, use consistent, logarithmically transformed real-world sizes of objects when drawing, imagining, and perceiving pictures. Although these effects were termed the canonical visual size by the authors, as they pointed out, their studies did not resolve the issue of whether canonical size is influenced by visual or conceptual information.

Because we not only perceive objects by looking at them, but to a large extent by touch, size information can also be represented spatially, in an amodal form (Loomis et al., 2013). Individuals differ in their preferred way of representing the world in their minds. Object visualizers primarily use vivid visual-like images. Spatial visualizers prefer schematic representations and their mental images depict spatial relations. Verbalizers mostly rely on analytical, verbal strategies when processing information (Blazhenkova \& Kozhevnikov, 2009). Both visual (object) images and spatial images contain information about the size of objects. The source of the visual imagery is visual information, the input of the spatial imagery may be visual, tactile, auditory or verbal information (Loomis et al., 2013). What is important, the touch can be a source of spatial information with regard not only to smaller objects but also to larger ones. For example, by touching a tree trunk, we can imagine its total height. That is why Kennedy (1993) argues that touch should be treated as a distal sense.

An increasing amount of research shows similarities between visual and haptic spatial processing (for an overview, see Lacey \& Sathian, 2014) which also applies to the size of objects. The accuracy of estimating the size of objects perceived by sight and by touch does not differ, especially when adults estimate the magnitude manually (Smith et al., 2005; Szubielska \& Bałaj, 2018). In sighted individuals both haptic and visual recognition are size-dependent (Craddock \& Lawson, 2009a, 2009b; Szubielska, 2015). In both a tactile domain and a visual domain, enlarging and reducing the size of objects in the mind increases the response time in imagery scaling task, which is a linear function of increasing the change of scale (Szubielska \& Bałaj, 2018; Szubielska \& Möhring, 2019).

So far research in a haptic domain did not investigate the issue of canonical size; while in the tactile domain (Woods et al., 2008), as earlier in the visual domain (Palmer et al., 1981), canonical views were shown. We still do not know 
"whether haptic representations store a canonical size for familiar objects" (Lacey \& Sathian, 2014, p. 6) and more specifically whether canonical size can be observed in embossed drawings. Importantly, vision control is not a requisite for drawing familiar objects, but drawings made under visual feedback are more recognizable and less erroneous than drawings made under haptic control (Vinter et al., 2018).

In the current study, we asked whether, similarly to the visual domain (Konkle \& Oliva, 2011), adults use canonical size in the tactile domain-when producing embossed drawings of familiar objects from memory. As there are similarities between visual and tactile processing of spatial features of objects (e.g., Lacey \& Sathian, 2014, Smith et al., 2005; Szubielska \& Bałaj, 2018) we expected that the canonical size effect reveals both in visual domain and in tactile drawings. Participants were asked to draw objects of various real-life sizes in two different conditions - using eyesight, on ordinary sheets of paper and, when blindfolded, using touch, on special foils designed for use by blind individuals to create drawings (the foil becomes convex in the place where the lines are made). The comparison of processing the size of objects in visual and tactile modality is interesting in the context of the concepts of a "metamodal" brain and multisensory object representation (e.g., Lacey \& Sathian, 2014, Pascual-Leone \& Hamilton, 2001). In our view, if the results were similar in both domains, it would mean that unisensory visual and haptic size processing leads to a shared spatial representation (Lacey \& Sathian, 2014; Loomis et al., 2013).

\section{METHOD}

\section{Participants}

This study included 17 participants ( 9 females) aged between 18 and 23 years $(M=21.29, S D=1.21)$. A priori power analyses using G-Power 3.1 yielded that 8 participants would be needed to detect a within-participants effect in a repeated measure analysis of variance (RM ANOVA), based on an effect size of $f=.83$ (cf. Konkle \& Oliva, 2011), significance levels of $p<.05$, and a power of .99. All participants declared normal or corrected to normal vision. Respondents were recruited from the university community and were MA students. Written informed consent was obtained from all individual participants included in the study prior to data collection. Eight participants (4 females) first drew on paper in the visual domain, and then on a special embossing foil in the tactile 
domain, and nine participants ( 5 females) first drew on special foil and next on paper.

\section{Materials}

Sheets of paper, pens, and special plastic sheets for embossed drawings (placed on a rubber mat) were used in the experiment. Sheets of paper and plastic sheets were in the A4 format $(210 \times 297 \mathrm{~mm})$.

\section{Procedure}

All procedures performed in studies involving human participants were in accordance with the ethical standards of the institutional and/or national research committee and with the 1964 Helsinki declaration and its later amendments or comparable ethical standards. The current study was approved by the Ethical Committee of the Institute of Psychology of the John Paul II Catholic University of Lublin.

The participants were randomly assigned to two experimental conditions. In the first condition, the participants first made drawings while blindfolded using a pen (which acted as a stylus) on a plastic sheet. They controlled the drawing performance with a non-dominant hand in this condition. They then drew on a sheet of paper, with their eyes open. In the second condition, the order of method of drawing was reversed.

The study was conducted in a single session and took approximately one hour. Participants took drawings on a given topic, sitting at the table. Each participant was given 16 sheets of paper and 17 plastic sheets (one extra sheet for familiarisation with embossed drawings by scribbling some lines and swirls). Participants had one minute to make each drawing in the visual domain and four minutes to make a single drawing in the tactile domain (which proved to be more than sufficient). In line with the study from Konkle and Oliva (2011), participants were instructed to draw one object per page. They were told that in this study we were not interested in their artistic skills. Not once did we mention to the participants that the size of the objects intrigues us, and any words connected with the size of objects were never used. The list of objects to draw contained 16 topics. The items on the list were assigned to 8 logarithmically increasing real-life sizes (two items per each size condition): (1) paperclip, key, (2) pet goldfish, apple, (3) hairdryer, running shoe, (4) backpack, computer monitor, (5) German shepherd dog, chair, (6) floor lamp, soda machine, (7) car, dump 
truck, (8) single-storey house, lighthouse. The order of items was randomised for each participant. Object Size Rank and Domain Factor (visual, tactile) were combined, amounting to a total of 32 trials.

The size of drawings was assessed by the authors of this paper. First, we drew a box on each sheet setting bounds around the object. In the case of drawings with extraneous objects (e.g., apple leaves, headlight beams of cars, etc.) we ignored the extraneous elements and drew the bounds over the target object. ${ }^{1}$ We then measured in centimeters the length of the diagonal of the box with a ruler. This length was treated as the drawn size of the objects (cf. Konkle \& Oliva, 2011).

\section{RESULTS}

A mixed analysis of variance (ANOVA) with the between-group factor of task order (visual first, tactile first) and the within-group factors of domain (visual, tactile) and object rank size (8) yielded no significant main effect of task order or of interactions between task order and other factors (all $F_{S}<1.40$, all $p s>.256$ ). Therefore, the data were collapsed across the task order factor, and then an ANOVA with repeated measurements was performed, which did not take into account the task order factor. Greenhouse-Geisser corrections were used to account for violations of the sphericity assumption whenever necessary.

The analysis yielded a significant effect of the domain factor, $F(1,16)=$ $=6.95, p=.018, \eta_{\mathrm{p}}{ }^{2}=.30$, observed power $=.70$ and object size rank factor, $F(3.55,56.79)=29.97, p<.001, \eta_{\mathrm{p}}{ }^{2}=.65$, observed power $=1.00$. These effects were qualified by a significant interaction between the domain factor and object size rank factor, $F(7,112)=3.77, p=.001, \eta_{\mathrm{p}}{ }^{2}=.19$, observed power $=.97 .^{2}$

Overall, drawings made in the visual domain were larger $(M=11.44$, $S D=4.21)$ than the ones made in the tactile domain $(M=10.36, S D=3.47)$; however, post-hoc comparisons using Bonferroni corrections showed that participants only produced significantly larger drawings in the visual condition than in the tactile condition when depicting objects of size rank of $6(p=.005)$, $7(p=.002)$ and $8(p=.005)$ (for means and $S D$ s, see Table 1). All other post-hoc comparisons were non-significant (all $p \mathrm{~s}>.059$ ).

\footnotetext{
1 This was done by the first and second author of the present paper. They identified that 30 percent of drawings included extraneous objects.

${ }^{2}$ A similar ANOVA calculated after filtering out the drawings which contained extraneous objects revealed similar effects.
} 
To explore the interaction further, similar ANOVAs for each domain condition were computed separately. Significant main effects of object size rank were found for a visual domain, $F(3.53,56.52)=27.15, p<.001, \eta_{\mathrm{p}}{ }^{2}=.63$, observed power $=1$ and a tactile domain, $F(3.83,61.23)=23.49, p<.001, \eta_{\mathrm{p}}^{2}=.60$, observed power $=1$. These significant effects of size rank of the object were best explained by a linear function both for the visual domain, $F(1,16)=73.44$, $p<.001, \eta_{\mathrm{p}}{ }^{2}=.82$, observed power $=1$ and tactile domain, $F(1,16)=58.64$, $p<.001, \eta_{\mathrm{p}}^{2}=.79$, observed power $=1$ indicating that drawing size increased linearly with increasing object size rank in both conditions.

Table 1. Drawn Size of Objects (Centimeters) as Function of Domain Factor and Object Size Rank. Standard Deviations Given in Parentheses

\begin{tabular}{ccc}
\hline \multirow{2}{*}{ Object Size Rank } & \multicolumn{2}{c}{ Domain } \\
\cline { 2 - 3 } & Visual & Tactile \\
\hline 1 & $7.95(1.15)$ & $7.29(0.83)$ \\
2 & $9.57(1.13)$ & $8.29(0.79)$ \\
3 & $9.08(1.12)$ & $8.83(0.97)$ \\
4 & $10.24(1.17)$ & $10.26(1.08)$ \\
5 & $11.32(1.15)$ & $10.66(1.10)$ \\
6 & $12.58(1.02)$ & $10.73(0.82)$ \\
7 & $15.81(1.28)$ & $13.96(1.08)$ \\
8 & $14.96(1.08)$ & $12.87(0.86)$ \\
\hline
\end{tabular}

\section{DISCUSSION}

The objective of the present study was to determine if canonical size can be observed in a tactile domain when undertaking the task of producing embossed drawings. To date, the canonical size effect has been shown exclusively in the visual domain - sighted adults used consistent, logarithmically transformed realworld sizes of objects in drawing, imagery, and perception tasks (Konkle \& Oliva, 2011). In the conceptual level, we were interested if unisensory visual and tactile processing leads to a shared spatial representation-either multimodal (Lacey \& Sathian, 2014) or amodal (Loomis et al., 2013). We predicted that the canonical size effect is present in both visual and tactile domain. Our results indicated a similarity between the tactile and visual domains which referred to the size of drawings of real-life objects. In both domains, the size of drawings 
increased linearly with the logarithm of the physical size of real-world objects. This demonstrates that canonical size is revealed not only in tasks in which visual perception is used (cf., Konkle \& Oliva, 2011), but also in those where tactile perception is engaged. Moreover, this suggests that spatial imagery is not modality-specific.

Despite this similarity, there were also differences between the visual and tactile conditions. Drawings of objects with the highest physical size rank (i.e., 6, 7 and 8) were bigger in the visual domain than in the tactile domain. These differences can be due to the fact that when blindfolded participants wanted to control the drawing produced using a dominating hand with their non-dominant hand. Many practitioners who design tactile graphics for blind individuals consider that "tangible display should be kept within a hand span - the distance from the end of the thumb to the end of the little finger when extended" (Edman, 1992, p. 105). With regard to the drawings of smaller real-world objects (with a physical size rank of 5 or less), there were no differences in the drawn size between the visual and tactile domains because smaller embossed drawings could be covered with one hand.

This study shows that canonical size can be disclosed both in the visual and tactile domain - which supports the conclusions about there being similarities in information processing for both visual and tactile size (cf. Craddock \& Lawson, 2009a, 2009b; Smith et al., 2005; Szubielska, 2015; Szubielska \& Bałaj, 2018), but it does not settle whether physical size information is represented both visually and tactilely. It is possible that in a tactile condition, participants used visual images and visualized drawings on sheets before they made them with the perceptual haptic feedback. On the other hand, the drawings were simplified and quite similar to each other when participants made them in the condition of sight and touch (what we observed when assessing their size-see Figure 1). Schematicity and repeatability of drawings made in the traditional way and in the blindfolded condition may indicate the use of the same amodal mental spatial representations when drawing under the control of vision and touch (cf. Blazhenkova \& Kozhevnikov, 2009 vs. Loomis et al., 2013). To answer the question which of these interpretations is more likely, it is worth continuing studies of canonical size in the tactile domain with participants who are congenitally blind (compared with blindfolded sighted participants) to adjudicate if physical size information might be represented non-visually. 


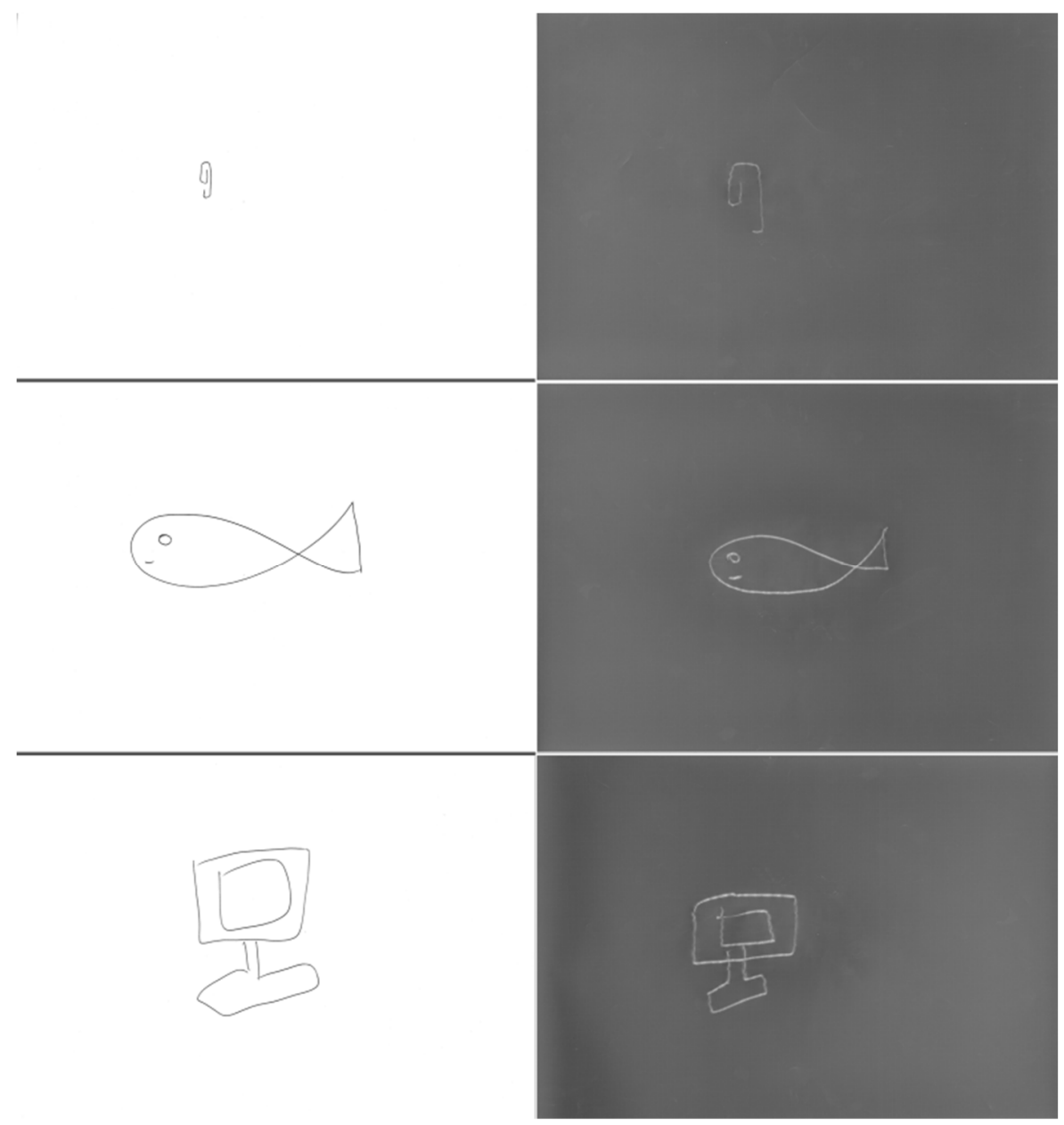

Figure 1. Sample Pairs of Drawings Made by Three Different Participants in Visual and Tactile Domain (Paper Clip, Goldfish, and Computer Monitor)

The present study has its strengths and limitations. We consider it a strength that, for the first time, we have manipulated the perceptual domain in which drawings of objects of different physical size were made. A limitation of our study concerns the fixed size of the sheets on which the participants made drawings. Konkle and Oliva (2011) manipulated the size of sheets and showed that the size at which objects were drawn relied on paper sizes, whereas the relative size of the drawn object within its frame (defined by the edges of the sheet) was similar across different sheet sizes. 
Overall, this is the first study investigating canonical size in the tactile domain. We have found that both in the visual and tactile domain, the drawn size of familiar objects increased linearly with the logarithmically increasing transformed physical size of these objects, suggesting that the canonical size can be observed both in the visual and tactile tasks. Future work with congenitally blind individuals may elucidate whether canonical size is visual in nature and if the physical size can be represented in the haptic domain. We also think that to make subsequent studies on canonical size more comparable, a precise list of extraneous objects should be created for each particular drawing topic. Moreover, it is worth to control cognitive style (that is, whether sighted participants are more object visualizers or spatial visualizers, see Blazhenkova \& Kozhevnikov, 2009) in future studies.

\section{REFERENCES}

Blazhenkova, O., \& Kozhevnikov, M. (2009). The new object-spatial-verbal cognitive style model: Theory and measurement. Applied Cognitive Psychology, 23, 638-663. https://doi.org/10.1002/acp.1473

Craddock, M., \& Lawson, R. (2009a). Size-sensitive perceptual representations underlie visual and haptic object recognition. PLoS One, 4, e8009. http://dx.doi.org/10.1371/journal.pone. 0008009

Craddock, M., \& Lawson, R. (2009b). The effects of size changes on haptic object recognition. Attention, Perception, \& Psychophysics, 71, 910-923. http://dx.doi.org/10.3758/APP.71.4.910

Edman, P. K. (1992). Tactile graphics. AFB Press.

Kennedy, J. M. (1993). Drawing and the blind: pictures to touch. Yale University Press.

Konkle, T., \& Oliva, A. (2011). Canonical visual size for real-world objects. Journal of Experimental Psychology: Human Perception and Performance, 37, 23-37. http://dx.doi.org/10. $1037 / \mathrm{a} 0020413$

Konkle, T., \& Oliva, A. (2012a). A familiar-size Stroop effect: Real-world size is an automatic property of object representation. Journal of Experimental Psychology: Human Perception and Performance, 38, 561-569. http://dx.doi.org/10.1037/a0028294

Konkle, T., \& Oliva, A. (2012b). A real-world size organization of object responses in occipitotemporal cortex. Neuron, 74, 1114-1124. http://dx.doi.org/10.1016/j.neuron.2012.04.036

Lacey, S., \& Sathian, K. (2014). Visuo-haptic multisensory object recognition, categorization, and representation. Frontiers in Psychology. https://doi.org/10.3389/fpsyg.2014.00730

Long, B., Konkle, T., Cohen, M. A., \& Alvarez, G. A. (2016). Mid-level perceptual features distinguish objects of different real-world sizes. Journal of Experimental Psychology: General, 145, 95-109. http://dx.doi.org/10.1037/xge0000130

Loomis, J. M., Klatzky, R. L., \& Giudice, N. A. (2013). Representing 3D space in working memory: Spatial images from vision, hearing, touch, and language. In S. Lacey \& R. Lawson (Eds.), Multisensory Imagery (pp. 131-155). Springer. https://doi.org/10.1007/978-1-46145879-1_8 
Palmer, S. E., Rosch, E., \& Chase, P. (1981). Canonical perspective and the perception of objects. In J. Long \& A. Baddeley (Eds.), Attention and performance IX (pp. 135-151). Erlbaum.

Pascual-Leone, A., \& Hamilton, R. H. (2001). The metamodal organization of the brain. Progress in Brain Research, 134, 427-445. https://doi.org/10.1016/S0079-6123(01)34028-1

Smith, M., Franz, E. A., Joy, S. M., \& Whitehead, K. (2005). Superior performance of blind compared with sighted individuals on bimanual estimations of object size. Psychological Science, 16, 11-14. https://doi.org/10.1111/j.0956-7976.2005.00773.x

Szubielska, M. (2015). Mental majorization of figures tactilely explored by sighted and congenitally blind individuals. Roczniki Psychologiczne, 18, 121-132. https://doi.org/10. 18290/rpsych.2015.18.1-5en

Szubielska, M., \& Bałaj, B. (2018). Mental size scaling of three-dimensional objects perceived visually or tactilely. Advances in Cognitive Psychology, 14, 139-149. https://doi.org/10. 5709/acp-0245-5

Szubielska, M., \& Möhring, W. (2019). Adults' spatial scaling: evidence from the haptic domain. Cognitive Processing, 20(4), 431-440. https://doi.org/10.1007/s10339-019-00920-3

Vinter, A., Bonin, P., \& Morgan, P. (2018). The severity of the visual impairment and practice matter for drawing ability in children. Research in Developmental Disabilities, 78, 15-26. https://doi.org/10.1016/j.ridd.2018.04.027

Woods, A. T., Moore, A., \& Newell, F. N. (2008). Canonical views in haptic object perception. Perception, 37, 1867-1878. http://dx.doi.org/10.1068/p6038 